\title{
Imaging blunt traumatic aortic injury: a Canadian tertiary care trauma centre case series
}

\author{
Christopher B. Lightfoot ${ }^{A}$, John M. Tallon ${ }^{B}$, Robert J. Abraham ${ }^{\mathrm{C}}$
}

Few individuals survive blunt traumatic aortic injury (TAI); however, the mortality rate is dramatically lowered by timely diagnosis and treatment. We performed a retrospective review of patients that had undergone aortography or were entered in a trauma database with a diagnosis of TAl between December 1, 1996, and April 1, 2004. The diagnostic utility of computed tomography (CT) was compared to the gold standards of aortography and operative findings. Forty charts were reviewed. Fifty-two percent had indirect CT signs and 39 percent had direct CT signs of TAI. Six TAl's were surgically confirmed and repaired. Computed tomography for TAI had a sensitivity of 100 percent and a specificity of $\mathbf{7 4}$ percent. Computed tomography imaging has obviated aortography in screening for TAI. Aortography continues to have a valuable diagnostic role prior to surgical management.

(DMJ 2006; 34(1); 31-36)

$\mathrm{B}$ lunt traumatic aortic injuries (TAI) are seen in 0.5 to 2 percent of chest trauma patients. ${ }^{1,2}$ The majority of these are the result of motor vehicle collisions. ${ }^{3}$

Blunt traumatic aortic injuries carry a very high mortality rate. Approximately 85 percent of patients die prior to hospital arrival. ${ }^{4}$ Nevertheless, of those that make it to hospital, 70 percent survive. Survival rate is dramatically affected by timely diagnosis and treatment. ${ }^{5,6}$

Imaging to evaluate TAI includes chest radiograph, trans-thoracic or trans-esophageal echocardiography, computed tomography (CT), and thoracic aortography. ${ }^{5}$ All are suggested to be appropriate in specific clinical situations. The use of these imaging modalities as initial screening tools is still debated. ${ }^{5}$ Thoracic aortography remains the "gold standard" for diagnosis from an imaging perspective, with operative findings (and/or autopsy findings) serving as the final gold standard. ${ }^{7}$ However, aortography is invasive, expensive, and time-consuming as a screening tool and advancing technology has pushed CT to the forefront as a screening and diagnostic tool. ${ }^{8-11}$

The purpose of this review was to evaluate the accuracy of CT screening for TAI at a Canadian tertiary care trauma centre. Our institution used a single-slice conventional $\mathrm{CT}$ and a single-slice helical CT during the study period. We hypothesized that the sensitivity and specificity of CT for TAI would equal the gold standard in imaging (aortography) with this level of CT technology.

\section{Materials and Methods}

Following research ethics board approval (Capital Health Ethics Review Board), we performed a retrospective review of patients screened for TAI. All patients that had undergone aortography or were entered in the provincial Trauma Registry that sustained a TAI from blunt thoracic trauma and were treated at the sole adult tertiary care trauma centre (QEII) between December 1, 1996, and April 1, 2004, were included (age $>15$ years). The Department of Radiology records were reviewed to identify all patients that had undergone aortography. Query fields for the EHS database

All Authors'Affiliation: Dalhousie University, Halifax, Nova Scotia, Canada

${ }^{A}$ Department of Diagnostic Radiology

${ }^{B}$ Departments of Emergency Medicine and Surgery

${ }^{c}$ Department of Diagnostic Radiology, Vascular and Interventional Radiology 
included "Aortic Injury" and "Blunt Thoracic Trauma". Patients that did not arrive alive at the hospital were excluded.

Screening for TAI in patients that had sustained blunt thoracic trauma was not standardised throughout the study period. The level of investigation was based on mechanism of injury and the clinical judgement of the trauma team members. The use of chest radiograph and $\mathrm{CT}$ varied and aortography was typically used to clarify or confirm a diagnosis.

Computed tomography scanning for TAI was performed with a conventional single-slice CT scanner for one case in our dataset. The institution was then upgraded to a single-slice helical model and subsequent CT's for TAI were performed on this unit. Three scans were performed on CT scanners at external institutions (two in Charlottetown, Prince Edward Island and one in Truro, Nova Scotia). The protocol for the QEII single-slice helical CT was a field of view from 3 $\mathrm{cm}$ above the aortic arch to iliac bifurcation with a slice thickness of $5 \mathrm{~mm}$ and a pitch of 2 . A total of $150 \mathrm{ml}$ of Isovue 300 contrast was injected at $2 \mathrm{ml}$ per second with a 45 second preparation delay.

Demographic information (age, sex, mechanism of injury), imaging results, operative management, and outcomes were evaluated. Data was collated using Microsoft Excel.

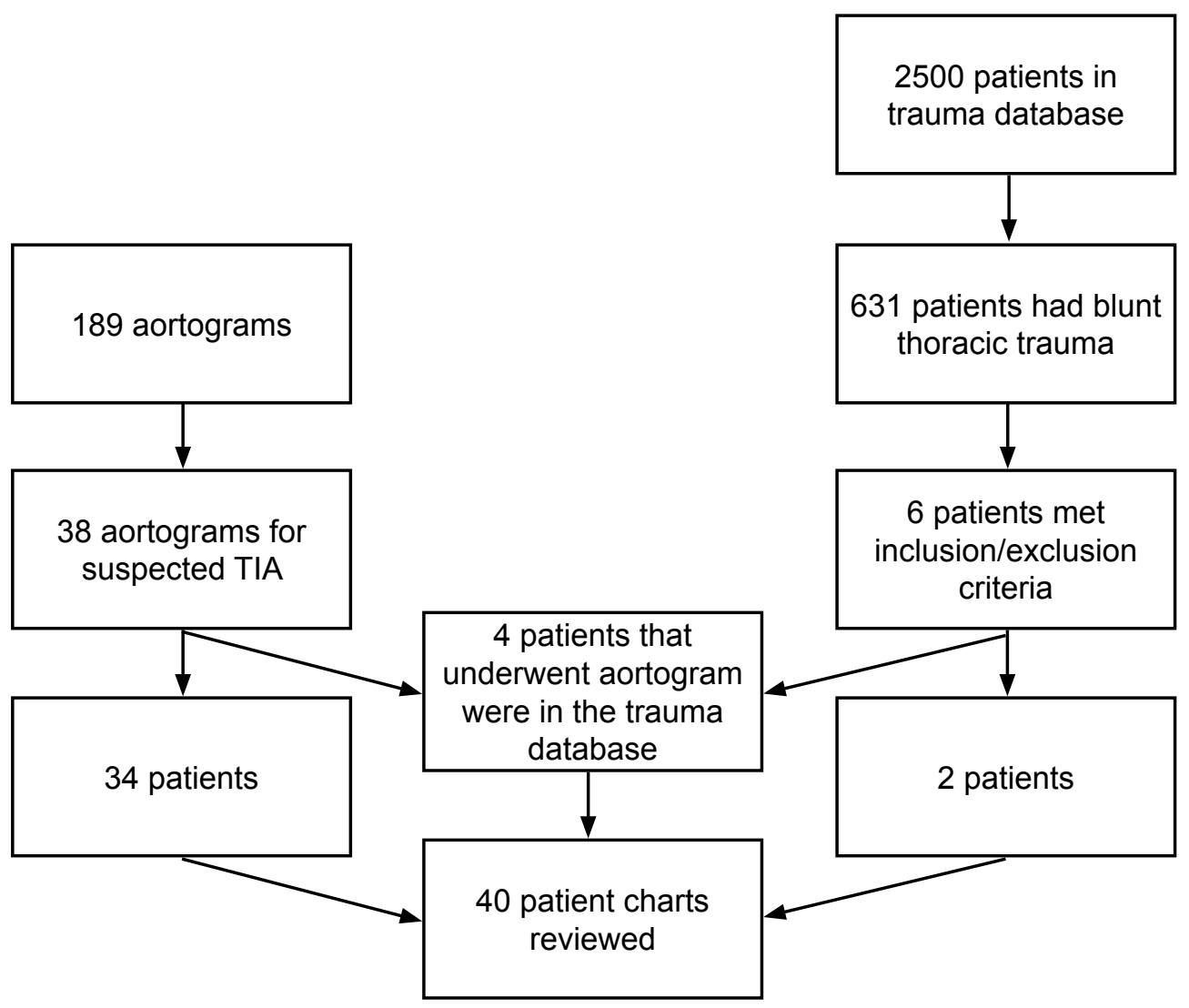

Figure 1. Flow chart of patient selection 


\section{Results}

The provincial Trauma Registry database contained approximately 2500 patients overall for the study period. Of these, 631 had sustained blunt thoracic trauma and six had a diagnosis of TAI and met our inclusion/exclusion criteria. ${ }^{12}$ A total of 189 aortograms were performed by the Department of Radiology during the study period. Through reviewing procedure reports, we determined that 38 were done for suspected TAI. Four patients from the Trauma Registry were among the 38 patients that had an aortogram.(Figure 1)

Forty patient charts were reviewed. Twentynine were male, and 11 were female. Mechanisms of injury included: 24 motor vehicle accidents, two all-terrain vehicle accidents, one motorcycle accident, one tractor roll-over, one pedestrian struck by a car, two falls from a significant height, three struck by falling trees, and two hit trees - one on a bicycle and one skiing. Mean age was 37 years with a range of 17 to 80 years. The average length

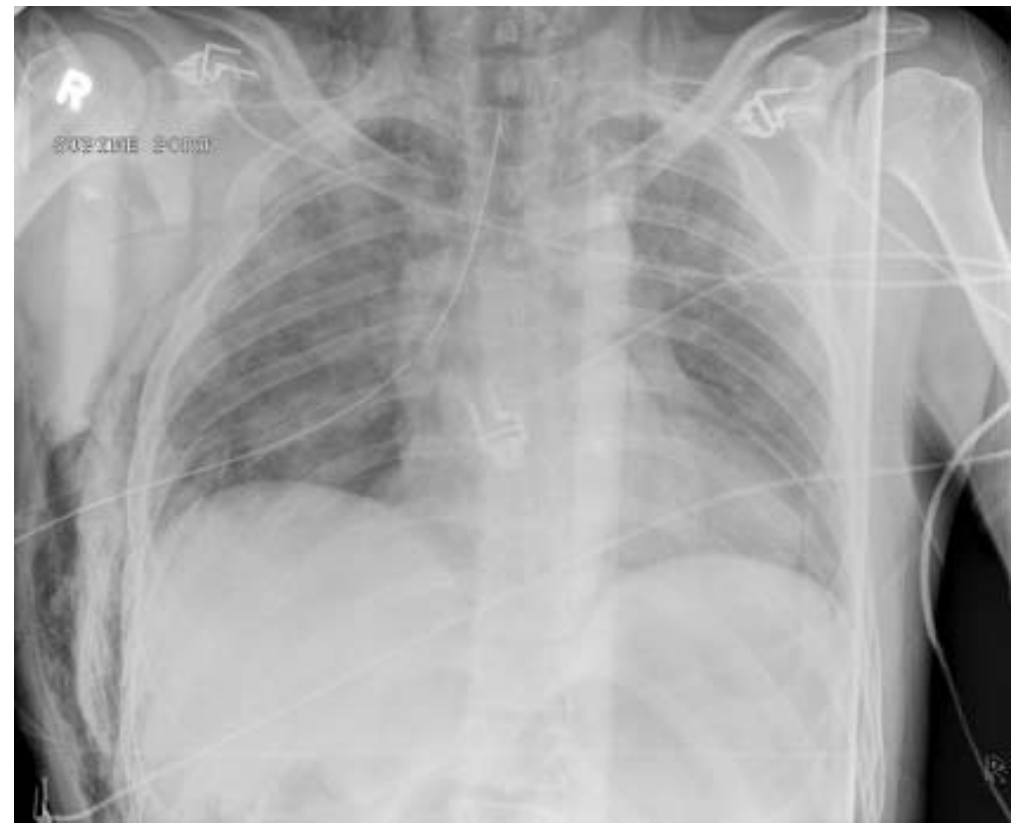

Figure 2. Chest X-ray of a 52-year-old male patient who was involved in a motor vehicle collision. Widening of the mediastinum and blurring of the aortic knuckle are seen. This study highlights the challenge of interpreting trauma films with immobilisation equipment artefact. of stay prior to discharge or transfer to local community hospital was 44 days. Six TAI's were surgically confirmed and repaired. Prior to surgery, two patients were diagnosed with $\mathrm{CT}$ alone, one was diagnosed with aortography alone, and three were congruently diagnosed with CT and aortogram. All six injuries were a result of motor vehicle accidents. Of these six, one patient died prior to discharge or transfer to local community hospital.

We were not able to locate chest radiographs on five of the 40 patients, three of which were later diagnosed with TAI. All radiographs were taken with a portable machine. Diagnostic accuracy of chest radiographs for TAI is presented.(Table 1) An example of a radiograph with findings suggestive of TAI is included.(Figure 2)

Of all the reviewed CT studies $(\mathrm{N}=33), 52$ percent had indirect $\mathrm{CT}$ signs (mediastinal hematoma) and 39 percent had direct CT signs (aortic wall irregularity or extravasation of contrast) of TAI. Seven diagnostic scans for TAI had subsequent negative aortograms. The sole examination on the conventional $\mathrm{CT}$ at the QEII showed indirect signs of TAI and had a subsequent normal aortogram. The external CT from Truro showed indirect signs of TAI and had a subsequent normal aortogram. One external CT from Charlottetown showed direct signs of TAI and had a subsequent normal aortogram. The second external CT from Charlottetown showed direct signs of TAI and was operated on without aortographic confirmation. 
Diagnostic accuracy of CT for TAI is presented.(Table 1) An example of a CT with direct findings suggestive of TAI is included.(Figure 3) An example of an aortogram with findings suggestive of a TAI is included.(Figure 4)

One patient that had indirect signs of TAI on CT was found to have a pseudoaneurysm of the proximal innominate artery with no active extravasation of contrast during aortography. She was managed conservatively by the surgical team and therefore was not included in our TAI group.

\section{Discussion}

We have determined that conventional and helical CT scanning throughout the study period has been 100 percent sensitive for TAI. However, our dataset had seven cases where imaging showed wall irregularity or extravasation of contrast with subsequent negative aortogram; thus confirming the role of aortography prior to surgical management.

Our findings are in keeping with others in the literature. Radiography is an intrinsic part of the early evaluation of the trauma patient. Radiographic findings suggestive of TAI include: a wide mediastinum, loss of the aortopulmonary window, aortic knob contour abnormalities, shift of the trachea, apical capping, displaced left mainstem bronchus, wide paraspinal lines, and rib injury. ${ }^{5}$ However, radiographic signs have been unreliable for screening of TAI's. ${ }^{13}$ This finding is reinforced by our study as not all patients with TAI had radiographic signs of acute injury.

Conventional CT has been shown adequate for screening with high sensitivity, but has been determined inadequate for definitive diagnosis of TAI. 14-16 In contrast to previous studies ${ }^{15,16}$, we had no false negatives in our small dataset.

Helical CT has been touted as being sufficient for screening and for definitive diagnosis of TAI. ${ }^{17-}$ ${ }^{20}$ Many have demonstrated a greater than 99 percent negative predictive value for TAI by helical CT, eliminating the need for many unnecessary aortograms ${ }^{17-20}$. However, equivocal cases on single detector helical CT still require aortography. ${ }^{17,21}$ Our study supports this assertion and suggests that despite direct imaging findings of TAI on singleslice CT, roughly 1 in 5 of these patients $(21 \%$ in this series) will have negative aortograms and not require surgical intervention.

Several of the CT examinations that showed indirect evidence of TAI indicated motion artefact compromised the images. With newer generation multi-slice detectors, the specificity of CT will almost certainly improve. A recent study by Melton et al. ${ }^{9}$ has examined the evolution of diagnostic imaging of TAI with changing CT technology. 
They have concluded that multidetector CT has the potential to entirely replace aortography for the screening, diagnosis and pre-operative evaluation of TAI. Obviously, further prospective analysis would be required before this can be recommended as the definitive diagnostic tool.

Computed tomography screening did not affect patient management in this series as decisions for surgical intervention were almost exclusively based upon aortographic findings, with only two exceptions. One of the patients in our study period died in hospital. A dramatic reduction in hospital arrival to surgery time has been observed when surgical management was based on CT results alone. ${ }^{21}$ Multiple-slice helical CT may reduce the demand for aortography and thus reduce time to surgical intervention and improve outcomes of TAI.

Limitations of this study include its retrospective nature. As we reviewed existing imaging reports from numerous radiologists, subjectivity in the interpretation of imaging studies cannot be excluded. Not all patients had identical screening for TAI. It should be noted that there may have been a number of individuals during our study period that were screened for TAI by chest radiogram and/or CT alone and did not undergo aortography. Therefore, our results may not extrapolate to a more generalised population. We used aortography as our gold standard. Some literature has shown very low rates of false-negative and false-positive aortograms for TAI. . $^{5,722,23}$ False-negatives are typically related to incomplete series or inadequate injec- tions. False-positives are typically related to ductal diverticulum, atheromatous plaques, or contrast streaming artefact. Lastly, the number of patients screened and treated for TAI at our institution was low.

In conclusion, CT imaging has superseded aortography in screening for TAI in this series of patients. A portion of those screened with singleslice helical CT require aortography for definitive diagnosis prior to surgical management. In the future, with more widespread availability of multipleslice helical CT, TAI outcomes may improve.

\section{References}

1. Frick EJ, Cipolle MD, Pasquale MD, et al. Outcome of blunt thoracic aortic injury in a level I trauma center: an 8-year review. Trauma 1997; 43:844-851.

2. Mirvis SE. Major vascular injury in trauma: in-

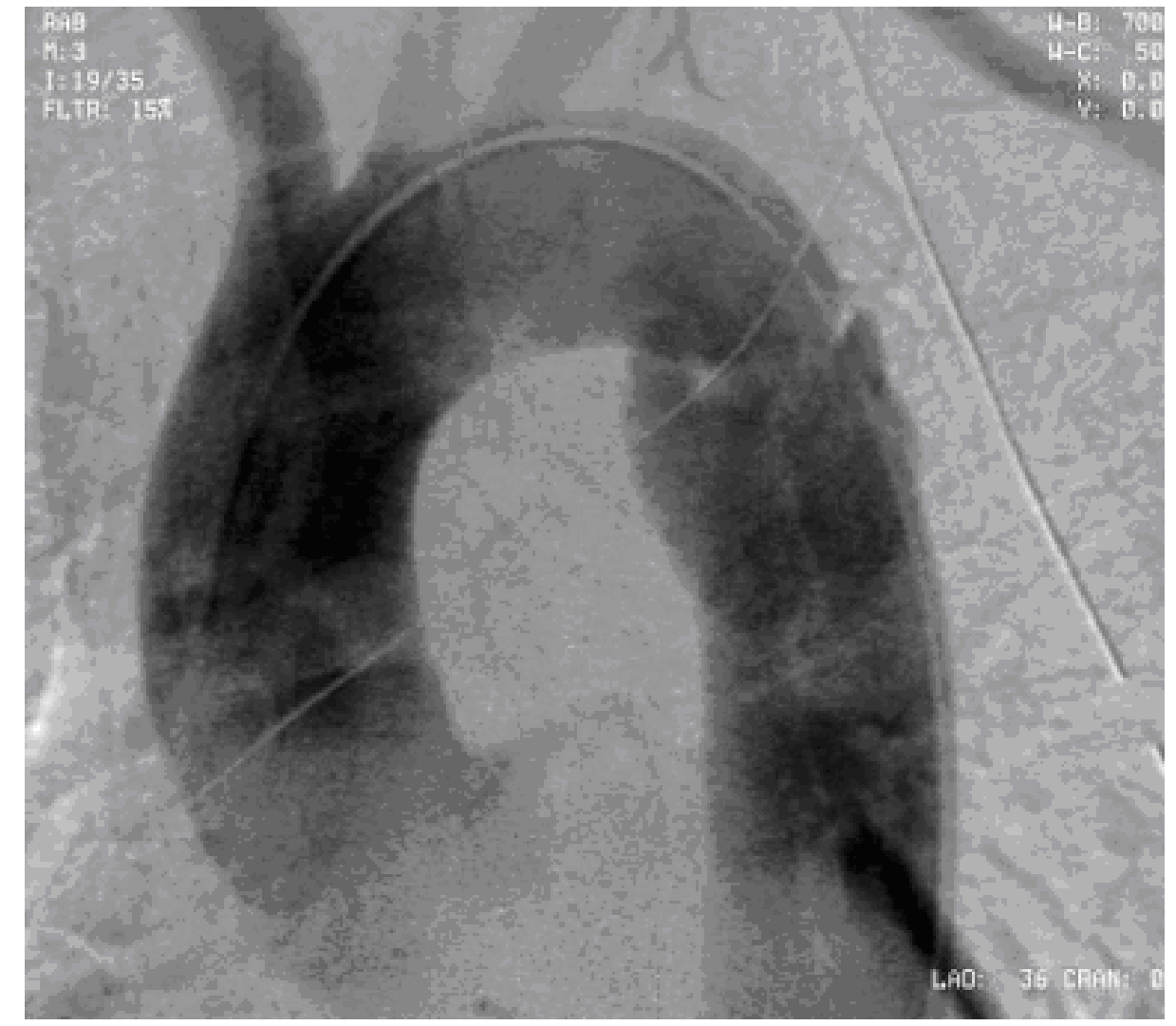

Figure 4. Aortogram of a 47-year-old male patient who was involved in a motor vehicle collision. Focal aortic rupture and pseudo-aneurysm formation below the isthmus of the proximal descending thoracic aorta with no frank extravasation is seen. 
fluence of new technology. Eur Radiol 1997; 7 (Suppl):278.

3. Williams JS, Graff JA, Uku JM, et al. Aortic injury in vehicular trauma. Ann Thorac Surg 1994; 57:726-30.

4. McGwin G, Reiff DA, Moran SG, et al. Incidence and characteristics of motor vehicle collision-related blunt thoracic aortic injury according to age. J Trauma 2002; 52:1-5.

5. Wintermark M, Wicky S, Schnyder P. Imaging of the acute traumatic injuries of the thoracic aorta. Eur Radiol 2001; 12:431-42.

6. Fabian TC, Richardson JD, Croce MA, et al. Prospective study of blunt aortic injury: multicenter trial of the American Association for the Surgery of Trauma. J Trauma 1997; 42:374-80.

7. Fishman,JE. Imaging of blunt aortic and great vessel trauma. J Thorac Imaging 2000;15(2):97-103.

8. Parker MS, Matheson TL, Rao AV, et al. Making the transition: the role of helical CT in the evaluation of potentially acute thoracic aortic injuries. AJR 2001; 176:1267-72.

9. Melton SM, Kerby JD, McGiffin D, et al. The evolution of chest computed tomography for the definitive diagnosis of blunt aortic injury: a singlecenter exprience. J Trauma 2004; 56:243-50.

10. Fabian TC, Davis KA, Favant ML, et al. Prospective study of blunt aortic injury: helical CT is diagnostic and antihypertensive therapy reduces rupture. Ann Surg 1998; 227:666-77.

11. Mirvis SE, Shanmuganathan K, Buell J, et al. Use of spiral computed tomography for the assessment of blunt trauma patients with potential aortic injury. J Trauma 1998; 45:922-30.

12. Nova Scotia Trauma Registry. http://www.gov. ns.ca/health/ehs/ (accessed June 3, 2005).

13. Mirvis SE, Bidwell JK, Buddemeyer EU, et al. Value of chest radiography in excluding traumatic aortic rupture. Radiology. 1987;163(2):487-93.

14. Raptopoulos V, Sheiman RG, Phillips DA, et al. Traumatic aortic tear: screening with chest CT. Radiology. 1992;182(3):667-73.

15. Durham RM, Zuckerman D, Wolverson M, et al. Computed tomography as a screening exam in patients with suspected blunt aortic injury. Ann Surg. 1994;220(5):699-704.

16. Hills MW, Thomas SG, McDougall PA, et al. Traumatic thoracic aortic rupture: investigation determines outcome. Aust N Z J Surg. 1994;64(5):312-8.
17. Fabian TC, Davis KA, Gavant ML, et al. Prospective study of blunt aortic injury: helical CT is diagnostic and antihypertensive therapy reduces rupture. Ann Surg. 1998;227(5):666-76..

18. Mirvis SE, Shanmuganathan K, Buell J, et al. Use of spiral computed tomography for the assessment of blunt trauma patients with potential aortic injury. J Trauma. 1998;45(5):922-30.

19. Pate JW, Gavant ML, Weiman DS, et al. Traumatic rupture of the aortic isthmus: program of selective management. World J Surg. 1999;23(1):59-63.

20. Wicky S, Capasso P, Meuli R, et al. Spiral CT aortography: an efficient technique for the diagnosis of traumatic aortic injury. Eur Radiol. 1998;8(5):828-33.

21. Downing SW, Sperling JS, Mirvis SE, et al. Experience with spiral computed tomography as the sole diagnostic method for traumatic aortic rupture. Ann Thorac Surg. 2001;72(2):495-501.

22. Ahrar K, Smith DC, Bansal RC, et al. Angiography in blunt thoracic aortic injury. J Trauma. 1997;42(4):665-9.

23. Johnson MS, Shah H, Harris VJ, et al. Comparison of digital subtraction and cut film arteriography in the evaluation of suspected thoracic aortic injury. J Vasc Interv Radiol. 1997;8(5):799-807. 